\title{
Analysis of the anticancer activity of curcuminoids, thiotryptophan and 4-phenoxyphenol derivatives
}

\author{
SHIREEN PARSAI ${ }^{1}$, RICK KECK ${ }^{1}$, EWA SKRZYPCZAK-JANKUN ${ }^{1}$ and JERZY JANKUN ${ }^{1-3}$ \\ ${ }^{1}$ Department of Urology, Urology Research Center, College of Medicine, University of Toledo, Toledo, OH 43614, USA; \\ ${ }^{2}$ Protein Research Chair, Department of Biochemistry, College of Sciences, King Saud University, Riyadh 11451, \\ Kingdom of Saudi Arabia; ${ }^{3}$ Department of Clinical Nutrition, Medical University of Gdańsk, Gdańsk 80-211, Poland
}

Received March 28, 2013; Accepted October 14, 2013

DOI: $10.3892 / \mathrm{ol} .2013 .1679$

\begin{abstract}
Curcumin, a non-nutritive yellow pigment derived from the rhizome of Curcuma longa (turmeric), is considered to be an established nutraceutical with anticancer activity. Turmeric contains three principal components, curcumin, demethoxycurcumin and bisdemethoxycurcumin, of which curcumin is most abundant and potent. The concurrence of a high consumption of turmeric and a low incidence of prostate cancer in Asian countries may suggest a role for curcumin in chemoprevention. Curcumin has been identified to exhibit anti-inflammatory, anti-oxidative and anticarcinogenic properties. Since the compound does not exhibit side effects, curcumin has been designated for several clinical trials as a treatment for human cancers. The pro-apototic, antioxidant and anti-inflammatory characteristics of curcumin are implicated in its anticancer activity, yet the mechanism of action of curcumin remains unknown. To achieve an effective pharmacological outcome, curcumin must reach and sustain appropriate levels at the site of action. However, the main disadvantage of curcumin is its high metabolic instability and poor aqueous solubility that limits its systemic bioavailability. To overcome this difficulty, the present study tested the anticancer activity of new curcumin-like compounds $(\mathrm{E} 21 \mathrm{cH}$ and Q012095H). Also, the use of new medicaments requires an understanding of their pharmacokinetic profiles and targets. Thus, molecular modeling methods were used to identify the targets of curcumin and curcumin-like compounds compared with other anticancer drugs (Q012138 and Q012169AT), which were used as the controls. The present study identified several enzymes that are targeted by curcumin, aldo-keto reductase family 1 member B10 (AKR1B10), serine/threonine-protein kinase, protein kinase $\mathrm{C}$, matrix metalloproteinase (MMP),
\end{abstract}

Correspondence to: Dr Jerzy Jankun, Department of Urology, Urology Research Center, College of Medicine, Health Science Campus, University of Toledo, 3000 Arlington Avenue, Toledo, $\mathrm{OH}$ 43614, USA

E-mail: jerzy.jankun@utoledo.edu

Key words: curcumin, thiotryptophan, 4-phenoxyphenol, derivatives, anticancer cyclooxygenase and epidermal growth factor receptor, which were tested as targets for these anticancer chemicals. All the examined small compounds demonstrated anticancer activity in the in vitro experiments and may impact cancer cells by acting on AKR1B10, MMP-9 and their targets.

\section{Introduction}

According to the American Cancer Society, prostate cancer is currently the second most common cause of cancer-related mortality among males. An estimated $>235,000$ new cases of prostate cancer are expected in the US during 2013 (1). Furthermore, in a recent study by Arcangeli et al, it is predicted that the increase in birth rate may correlate with an increased prevalence of prostate cancer in the United States by 2020 (2). However, with the establishment of diagnostic markers, including prostate-specific antigen screening, and recent advances in molecular imaging, clinicians are able to detect early cancer proliferation prior to the development of apparent clinical manifestations and, more significantly, prior to the occurrence of metastasis. This affords clinicians more time to design the appropriate and effective treatment procedures. The current treatment methods for prostate cancer include the administration of steroidal and non-steroidal anti-androgens, radiation therapy, chemotherapy, surgery or a combination of these modalities. Although these options may be successful in controlling the progression of prostate cancer, they are often associated with comorbidities that affect urinary and sexual function. Therefore the aim of prostate cancer research is to develop innovative treatment options to avoid such complications. Several characteristics of prostate cancer make it useful to serve as a model for developing new chemopreventive techniques, including its high prevalence, heterogeneous presentation, long latency, slow progression, preneoplastic lesions and tumor marker availability $(2,3)$.

Males have an equal rate of histological prostate cancer worldwide, as assessed by volume, grade and number of malignant foci (4). However, disease incidence varies widely according to the geographic location. Western nations have higher rates of mortality compared with Asian countries, including India, China and Japan. More notably, migrating populations from low-risk areas (Asian countries) to high-risk 
areas (Western countries) also have an increased risk of developing prostate cancer. Since genetic predisposition accounts for only $5-10 \%$ of cases, as cited by the American Cancer Society (1), the uniting theme in the literature has become identifying the environmental factors that promote or inhibit the development of prostate cancer.

Foods or part of foods with medicinal value, termed nutraceuticals, which are prepared and consumed variably across cultures, may be active in the prevention and treatment of diseases, including prostate cancer. Curcumin, a non-nutritive yellow pigment derived from the rhizome of Curcumin longa (turmeric), has received attention as an established nutraceutical that is capable of anticancer activity (5). Turmeric contains three principal components, curcumin, demethoxycurcumin and bisdemethoxycurcumin, of which curcumin is the most abundant and potent (6-9). The concurrence of a high consumption of turmeric in Asian countries and a low incidence of prostate cancer suggest its role in chemoprevention (10). Curcumin and a number of its derivatives have been identified to exhibit anti-inflammatory, antioxidative and anticarcinogenic properties (11). As the compound does not exhibit toxic, genotoxic or teratogenic properties, curcumin has been selected for several clinical trials to be used as a possible treatment for human cancers $(3,5,11)$. Curcumin has been shown to diminish the proliferation of androgen-dependent and androgen-independent prostate cancer cell lines (12). Furthermore, studies have revealed a wide array of therapeutic activities against multiple myeloma, pancreatic cancer, myelodysplastic syndromes, colon cancer, psoriasis, Alzheimer's disease and others (13). The pro-apototic, antioxidant and anti-inflammatory properties of curcumin are implicated in its anticancer activity, yet the mechanism of action of curcumin remains unknown (8). Curcumin is a highly pleiotropic molecule with multiple mechanisms by which it may mediate chemotherapy and chemopreventive effects on cancer, while remaining safe with little or no side effects. This dietary compound has been shown to inhibit several cell signaling pathways, including nuclear factor (NF)- $\kappa \mathrm{B}$, activating protein-1, tumor necrosis factor and metastatic and angiogenic pathways. The compound also inhibits certain enzymes, including cyclooxygenase (COX)-2 and matrix metalloproteinases (MMPs) $(9,13,14)$. The present study randomly identified several enzymes that are essential in carcinogenesis and are also targeted by curcumin, aldo-keto reductase family 1 member B10 (AKR1B10), serine/threonine-protein kinase (mTOR), protein kinase C (PKC), MMP-9, COX-1 and epidermal growth factor receptor (EGFR), to gain further insight into the mechanism of action $(5,7,13,15-17)$.

Curcumin has a poor systemic bioavailability as it is not able to reach and sustain the appropriate levels at the site of action due to its high metabolic instability and poor aqueous solubility $(18,19)$. The present study aimed to identify the anticancer activity of curcumin-like compounds with potentially greater bioavailability, and speculate the protein targets of these compounds that are implicated in the mechanism of action. Novel curcumin-like compounds, E21cH and Q012095H, with greater water solubility were tested. Molecular modeling methods were used to identify the targets of curcumin and curcumin-like compounds by comparing them with other anticancer drugs (Q012138 and Q012169AT), which were used as a controls.

\section{Materials and methods}

Compounds. The small molecular chemicals with anticancer activities were obtained from PharmaIP, LLC (Greenwich CT, USA). Curcumin [(1E,4Z,6E)-5-hydroxy-1,7-bis(4-hyd roxy-3-methoxy-phenyl)hepta-1,4,6-trien-3-one]; Q0121138 [4-[[(1S)-1-(benzothiophen-2-ylmethyl)-2-ethoxy-2-oxoethyl]carbamoyl]phenyl] methylammonium; Q012095H (1E,4Z,6E)-1,7-bis[5-(2-dimethylaminoethyl sulfanyl)-2-thie nyl]-5-hydroxy-hepta-1,4,6-trien-3-one; Q012138 [4-[[(1S)-1 -(benzothiophen-2-ylmethyl)-2-ethoxy-2-oxo-ethyl] carbamoyl] phenyl] methylammonium; and Q012169AT (N-ethyl-5-hydroxy-2-phenoxy-benzamide; Fig. 1). All the compounds were dissolved in dimethyl sulfoxide (DMSO) $2.5 \mathrm{mg} / \mathrm{ml}$ and stored at $-20^{\circ} \mathrm{C}$ until they were used.

Cell culture and clonal assay. The DU-145 human prostate cancer cell line was grown in RPMI-1640 medium supplemented with $10 \%$ fetal bovine serum (Atlanta Biologicals, Lawrenceville, GA, USA) and $100 \mathrm{U} / \mathrm{ml}$ penicillin, $100 \mu \mathrm{g} / \mathrm{ml}$ streptomycin (Sigma-Aldrich, St. Louis, MO, USA). A total of $\sim 50$ or 100 viable DU-145 cells (Trypan blue viability assay, two separate trials) were plated in 0.5 or $1 \mathrm{ml}$ of complete medium onto 12 or 24 -well tissue culture dishes. The cells were allowed to attach for $48 \mathrm{~h}$. The cells were then treated for $4 \mathrm{~h}$ with 1.2-, 2.5-, 5.0- or 10- $\mu$ l allotments of DMSO, curcumin, E21cH, Q0121138, Q012095H or Q012169AT dissolved in $1 \mathrm{mg} / \mathrm{ml}$ DMSO. The surviving cells were incubated for nine days to allow colony formation and then rinsed with $10 \%$ saline, fixed with $100 \%$ methanol and stained using Giemsa stain. The colony counts were performed under x10 magnification (Stereomaster, Thermo Fisher Scientific Inc., Waltham, MA, USA). The experiments were repeated in triplicate to determine the anticancer activity.

Molecular modeling. Two-dimensional structures (2D) of small molecular chemicals were created by AccelrysDraw v. 4.0 (Accelrys, Inc., San Diego, CA, USA) in an SKC format. The 2D structures were converted into three dimensional and PDB format files using a web-based program (http://www. molecular-networks.com/products). Docking of the potential inhibitors to the proteins was performed using VINA Autodock (Molecular Graphics Lab, The Scripps Research Institute, La Jolla, CA, USA) (20). The protein structures were downloaded from http://www.rcsb.org/pdb/home/home.do as: 1zua, AKR1B10 (21); 3oaw, mTOR (22); 1yrk, PKC (23); 2ovx, MMP-9 (24); 3ln1, COX-2 (25) and 2itx, EGFR (26). A search box was set up with following parameters: AKR1B10 human NADPH-dependent aldo-keto reductase (center: $\mathrm{x},-29 ; \mathrm{y}, 22$; z, 0.1; size: $\mathrm{x}, 50 ; \mathrm{y}, 50 ; \mathrm{z}, 50$ ), mTOR (center: $\mathrm{x},-17.5 ; \mathrm{y},-11$; z, -12; size: x, 50; y, 40; z, 46). PKC (center: x, 25; y, 40; z, 31; size: $\mathrm{x}, 40 ; \mathrm{y}, 44 ; \mathrm{z}, 74)$, MMP-9 (center: $\mathrm{x}, 27 ; \mathrm{y}, 6$; z, 51; size: $\mathrm{x}, 40 ; \mathrm{y}, 56 ; \mathrm{z}, 40), \mathrm{COX}-2$ (center: $\mathrm{x}, 32 ; \mathrm{y},-22 ; \mathrm{z},-16$; size: $\mathrm{x}, 40 ; \mathrm{y}, 40 ; \mathrm{z}, 40$ ) and EGFR (center: $\mathrm{x},-47 ; \mathrm{y},-2 ; \mathrm{z},-22$; size: $\mathrm{x}, 50 ; \mathrm{y}, 40 ; \mathrm{z}, 50)$. The inhibitors that were present in the PDB structures were used to determine the center of the search and later removed from structure. The small molecules were kept flexible by allowing rotation around the single bonds. By default, VINA Autodock analyzes eight various protein/inhibitor complexes (conformers) and the one with the 


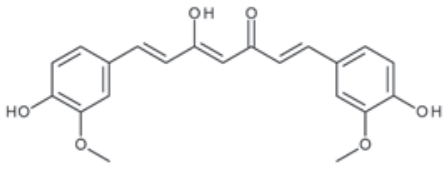

curcumin

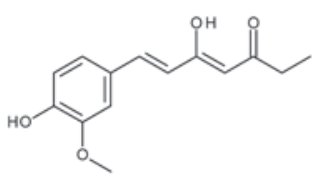

$\mathrm{E} 21 \mathrm{cH}$

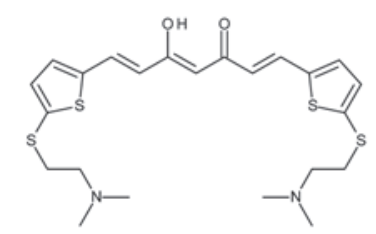

Q012095H

Figure 1. Structure of compounds with anticancer activities. Curcumin and curcumin-like compounds are presented in the enol form, which is more thermodynamically stable (60).

lowest free energy is considered the most probable. Free energy is converted to $K_{\mathrm{i}}$ using the following formula (20,27-30): $\mathrm{K}_{\mathrm{i}}=\exp [\Delta \mathrm{G} /(\mathrm{R} \times \mathrm{T})]$, where $\Delta \mathrm{G}$ is Gibbs free energy change, $\mathrm{R}$ is the gas constant and $\mathrm{T}$ is the absolute temperature. The final analyses of structures that were generated by Autodock and the generation of the figures was performed using PyMOL v. 1.4 (Schrödinger, München, Germany) (31,32).

\section{Results}

In vitro anticancer activity. The present study tested the anticancer activities of $>30$ curcuminoids, thiotryptophanes and 4-phenoxyphenol derivatives. In the clonal assay, Q012095H demonstrated the strongest anticancer activity, followed by Q012138 and Q012165H. E21cH and curcumin activity were comparable with each other but lower than the three others (Fig. 2). The highest concentrations of Q012095H and Q012138 showed a complete inhibition of cancer cell growth.

Molecular modeling. The results of the docking are illustrated in Fig. 3 and the calculated $\mathrm{K}_{\mathrm{i}}$ values are provided in Table I. All the compounds that were tested contained an aldo-keto moiety. One of the human enzymes that was tested in the in silico experiment was AKR1B10, an NADPH-dependent aldo-keto reductase that reduces a variety of aldehydes and ketones. AKR1B10 has been reported to be upregulated in number of cancers. Additionally, AKR1B10-gene silencing results in the inhibition of colorectal cancer cell growth, suggesting that AKR1B10 regulates cell proliferation (33). It has been proposed that AKR1B10 controls retinoic acid signaling and impacts the carcinogenesis process. Also, tolrestat, which efficiently inhibits AKR1B10, is suggested to have a potential application in cancer control $(34,35)$. Thus, the investigated chemicals were tested for the capacity to bind to the active site of this enzyme. All the investigated chemicals were found to bind near the active site and functionally block its access. The calculated $\mathrm{K}_{\mathrm{i}}$ was in $\mu \mathrm{M}$ levels for all the tested chemicals, indicating their relative strength of affinity (Table I).

\section{Discussion}

mTOR, a serine/threonine protein kinase, regulates cell growth, cell proliferation, cell motility, cell survival, protein synthesis and transcription (36). The inhibition of mTOR mediates the antiproliferative effects of curcumin in numerous human and non-human cell lines $(15,37,38)$. In addition, curcumin has been reported to be able to dissociate the raptor subunit from mTOR as well as inhibit mTORC1 activity (15). Liu et al designed several idopyrimidinone (1) 4-methylpteridinones that bind to a small pocket within the mTOR binding site. This inhibitor in the protein structure was used as a center of search (22). In the present study, the molecular modeling revealed that all the compounds that were tested had a relatively low affinity and bound in various locations outside the active site of mTOR. Only E21cH and Q012095H were able to bind in proximity to where the inhibitor was localized. mTOR may be an unlikely target of the chemicals that were tested. By contrast, Lin et al stated that PKC and mTOR were the major upstream molecular targets for curcumin (39). A possible explanation is that the products of curcumin degradation act on mTOR instead of curcumin itself $(7,8,13,14,18,19,40)$.

Curcumin is an inhibitor of PKC. Consequently, curcumin inhibits the activation of $\mathrm{NF}-\kappa \mathrm{B}$ and the expression of oncogenes, including c-jun, c-fos, c-myc, NF- $\kappa \mathrm{B}$-inducing kinase (NIK), mitogen-activated protein kinases, ERK, ELK, phosphoinositide 3-kinase, Akt, cyclin-dependent kinases and inducible nitric oxide synthase (39). Conboy et al performed molecular modeling and identified that curcumin was able to dock effectively on PKC. However, curcumin did not directly inhibit PKC activity, but rather increased its degradation (41). The calculations in the present study revealed all tested compounds bind to PKC in essentially the same place, but with low affinity.

Traditionally, MMP-9 was associated with tumor angiogenesis and metastasis by lysing proteins of connective tissue $(42,43)$. However, curcumin has been reported to protect MMP-9 from proteolytic degradation (44). MMP-9 plays a critical function in normal and pathological angiogenesis and/or controlling the biological activity of growth factors, cytokines and chemokines (45). Proteolytic enzymes that stimulate angiogenesis and metastasis frequently show other functions in carcinogenesis in addition to their traditional roles (46-48). Ravindranath et al reported that blocking the activity of MMP-9 may arrest cell growth and proliferation in addition to the inhibition of invasion and angiogenesis (49). All the chemicals that were tested were observed to bind to the active site of MMP-9 in the proximity of the MMP-9 inhibitor (5-(4-phenoxy phenyl)-5-(4-pyrimidin-2-ylpiperazin-1-yl)pyrimidine-2,4,6(2H,3H)-trione) with high affinity.

Curcumin possesses anti-inflammatory activity and is a potent inhibitor of reactive oxygen generating enzymes, including COX $(15,37)$. Curcumin itself is a potent scavenger 
Table I. Calculated $\mathrm{K}_{\mathrm{i}}$ for the various protein and inhibitor complexes.

\begin{tabular}{lclllll}
\hline Compound & $\begin{array}{c}\text { AKR1B10 } \\
(\mathrm{kcal} / \mathrm{M}) / \mathrm{K}_{\mathrm{i}}(\mathrm{M})\end{array}$ & $\begin{array}{c}\mathrm{mTOR} \\
(\mathrm{kcal} / \mathrm{M}) / \mathrm{K}_{\mathrm{i}}(\mathrm{M})\end{array}$ & $\begin{array}{c}\mathrm{PKC} \\
(\mathrm{kcal} / \mathrm{M}) / \mathrm{K}_{\mathrm{i}}(\mathrm{M})\end{array}$ & $\begin{array}{c}\mathrm{MMP}-9 \\
(\mathrm{kcal} / \mathrm{M}) / \mathrm{K}_{\mathrm{i}}(\mathrm{M})\end{array}$ & $\begin{array}{c}\mathrm{COX}-2 \\
(\mathrm{kcal} / \mathrm{M}) / \mathrm{K}_{\mathrm{i}}(\mathrm{M})\end{array}$ & $\begin{array}{c}\text { EGFR } \\
(\mathrm{kcal} / \mathrm{M}) / \mathrm{K}_{\mathrm{i}}(\mathrm{M})\end{array}$ \\
\hline Curcumin & $-7.6 / 2.8 \times 10^{-6}$ & $-6.9 / 9.0 \times 10^{-6} \mathrm{NA}$ & $-6.2 / 2.9 \times 10^{-5}$ & $-9.1 / 2.2 \times 10^{-7}$ & $-7.3 / 4.6 \times 10^{-6} \mathrm{NA}$ & $-6.7 / 1.3 \times 10^{-5}$ \\
E21cH & $-7.4 / 3.9 \times 10^{-6}$ & $-5.8 / 5.7 \times 10^{-5}$ & $-4.9 / 2.6 \times 10^{-4}$ & $-7.9 / 1.7 \times 10^{-6}$ & $-7.6 / 2.8 \times 10^{-6}$ & $-6.3 / 2.5 \times 10^{-5}$ \\
Q012095H & $-5.8 / 5.7 \times 10^{-5}$ & $-4.6 / 4.3 \times 10^{-4}$ & $-4.5 / 5.1 \times 10^{-4}$ & $-6.2 / 2.9 \times 10^{-5}$ & $-6.1 / 3.5 \times 10^{-5} \mathrm{NA}$ & $-5.2 / 1.6 \times 10^{-4}$ \\
Q012138 & $-7.1 / 6.5 \times 10^{-6}$ & $-6.2 / 2.9 \times 10^{-5} \mathrm{NA}$ & $-5.8 / 5.7 \times 10^{-5}$ & $-8.9 / 3.1 \times 10^{-7}$ & $-9.2 / 1.9 \times 10^{-7}$ & $-6.2 / 2.9 \times 10^{-5}$ \\
Q012169AT & $-6.2 / 2.9 \times 10^{-5}$ & $-6.9 / 9.0 \times 10^{-6} \mathrm{NA}$ & $-4.9 / 2.6 \times 10^{-4}$ & $-7.8 / 2.0 \times 10^{-6}$ & $-8.8 / 3.7 \times 10^{-7}$ & $-6.6 / 1.5 \times 10^{-5}$ \\
\hline
\end{tabular}

AKR1B10, aldo-keto reductase family-1 member B10; mTOR, serine/threonine kinase; PKC, protein kinase C; MMP, matrix metalloproteinase; COX-2, cyclooxygenase-2; EGFR, epidermal growth factor receptor; NA, not applicable.

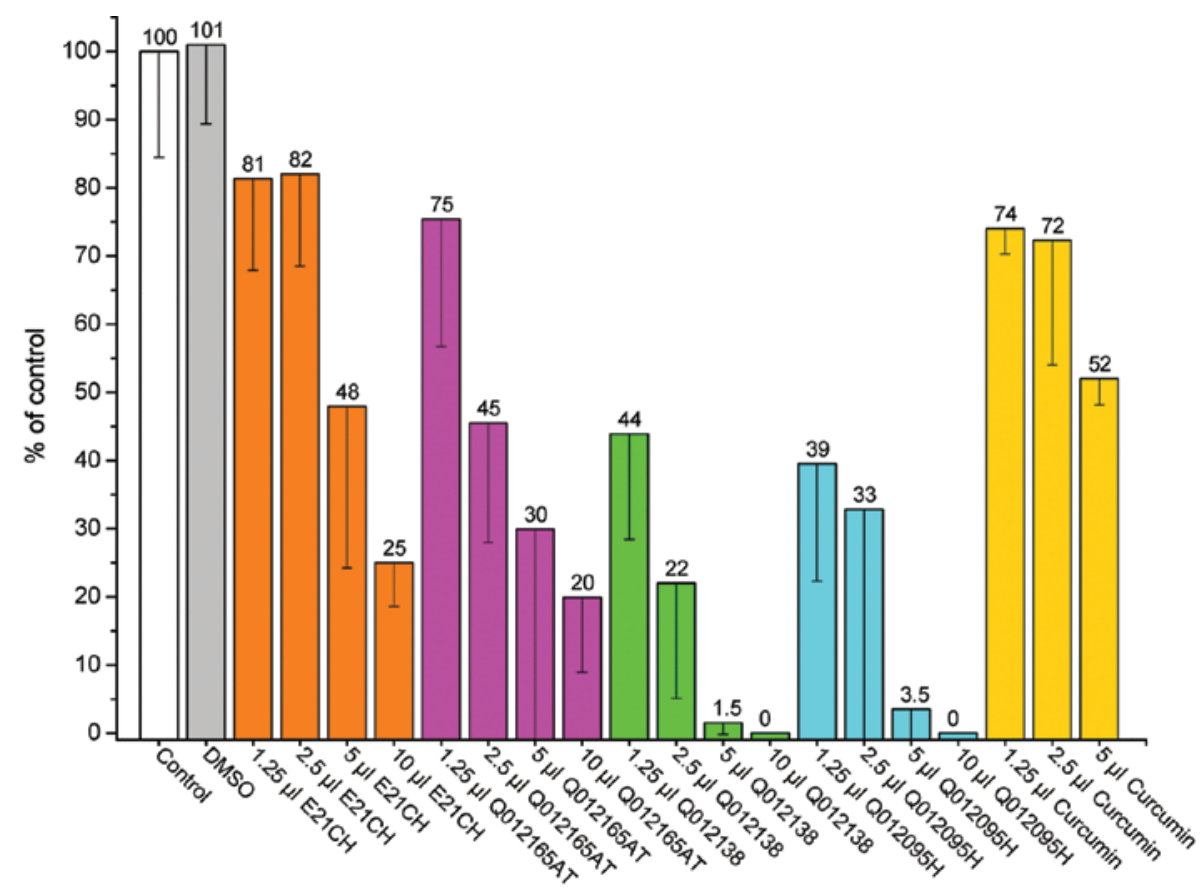

Figure 2. Survival of cancer cells treated with the chemicals that were tested depends on the concentration of the delivered compound in the cell media. DMSO, dimethyl sulfoxide.
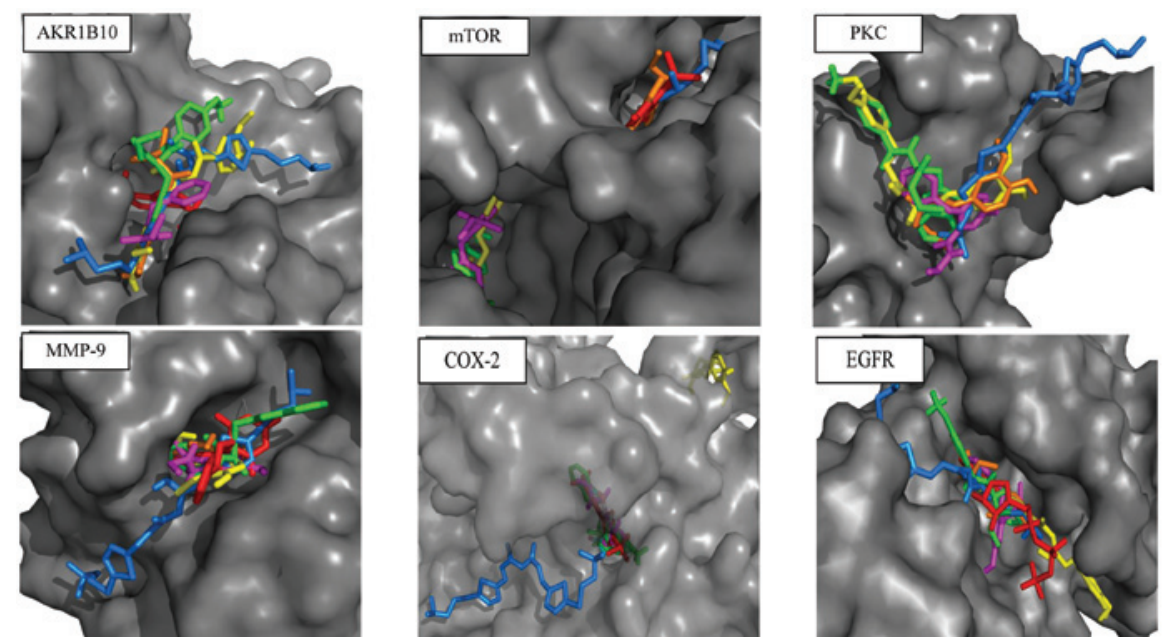

Figure 3. Surface model of the active sites of the enzymes that were tested. All the potential inhibitors are shown as stick models. Curcumin (yellow), E21cH (orange), Q012095H (blue), Q012138 (green) and Q012169AT (magenta). The inhibitor from the PDB structure is shown in red. AKR1B10, aldo-keto reductase family-1 member B10; mTOR, serine/threonine kinase; PKC, protein kinase C; MMP, matrix metalloproteinase; COX-2, cyclooxygenase-2; EGFR, epidermal growth factor receptor. 
of free radicals and the inhibition of COX potentiates its anticancer activity (50-52). Although the specific regulation of COX-2 by curcumin is not fully understood, the evidence suggests that curcumin regulates $\mathrm{COX}-2$ at the transcriptional and the post-translational levels $(17,53)$. In the present study, molecular modeling revealed that $\mathrm{E} 21 \mathrm{cH}$, Q012138 and Q012169AT bind to COX-2 with a high affinity, deep in the tunnel of the active site where celecoxib (4-[5-(4-methylpheny 1)-3-(trifluoromethyl)-1h-pyrazol-1-yl]benzenesulfonamide) is bound (25). However, Q012095H and curcumin were observed to bind outside of this site. This is in contrast with studies that state that curcumin inhibits COX (16,54-56). A possible explanation is that the non-enzymatic degradation of curcumin occurs, resulting in degradation products that are formed through cleavage of the heptadienone chain that connects the phenolic rings (57). Dong et al have shown that COX acts as a dimer, where one monomer with a heme moiety is active and the other is apo, which acts as the allosteric site, controlling activity of the active monomer (58). Thus, the docking scenario in its simplification in silico may not reflect the true situation in vivo, which may be more complex.

$\mathrm{Xu}$ et al reported that cyclohexanone analogs that are designed based on the curcumin structure are potential EGFR inhibitors and exhibit antiproliferative activity in human tumor cell lines. Cyclohexanone analogs fit in the active site of EGFR, as shown by molecular docking (59). This was confirmed by the experimental modeling in the present study. All the investigated compounds also bound the EGFR active site, but with low affinities.

Based on the results of the present study, AKR1B10 and MMP-9 have been shown to be the most likely targets of curcumin and curcumin-like derivatives. Curcumin and the investigated curcumin-like compounds bound the other proteins that were tested outside of the active site or with low affinities.

\section{Acknowledgements}

This study was supported in part by a grant from Frank Stranahan Endowed Chair.

\section{References}

1. American Cancer Society: Cancer Facts \& Figures 2013. http:// www.cancer.org/research/cancerfactsstatistics/index. Accessed March 24, 2013.

2. Arcangeli S, Pinzi V and Arcangeli G: Epidemiology of prostate cancer and treatment remarks. World J Radiol 4: 241-246, 2012.

3. Cimino S, Sortino G, Favilla V, et al: Polyphenols: key issues involved in chemoprevention of prostate cancer. Oxid Med Cell Longev 2012: 632959, 2012.

4. Fleshner $\mathrm{N}$ and Zlotta AR: Prostate cancer prevention: past, present, and future. Cancer 110: 1889-1899, 2007.

5. Agrawal DK and Mishra PK: Curcumin and its analogues: potential anticancer agents. Med Res Rev 30: 818-860, 2010.

6. Da-Lozzo EJ, Moledo RC, Faraco CD, Ortolani-Machado CF, Bresolin TM and Silveira JL: Curcumin/xanthan-galactomannan hydrogels: rheological analysis and biocompatibility. Carbohydr Polym 93: 279-284, 2013.

7. Hsu CH and Cheng AL: Clinical studies with curcumin. Adv Exp Med Biol 595: 471-480, 2007.

8. Park W, Amin AR, Chen ZG and Shin DM: New perspectives of curcumin in cancer prevention. Cancer Prev Res (Phila) 6: 387-400, 2013.

9. Strimpakos AS and Sharma RA: Curcumin: preventive and therapeutic properties in laboratory studies and clinical trials. Antioxid Redox Signal 10: 511-545, 2008
10. Lin L, Shi Q, Nyarko AK, et al: Antitumor agents. 250. Design and synthesis of new curcumin analogues as potential anti-prostate cancer agents. J Med Chem 49: 3963-3972, 2006.

11. Mimeault M and Batra SK: Potential applications of curcumin and its novel synthetic analogs and nanotechnology-based formulations in cancer prevention and therapy. Chin Med 6: 31, 2011.

12. Dorai T, Gehani N and Katz A: Therapeutic potential of curcumin in human prostate cancer-I. curcumin induces apoptosis in both androgen-dependent and androgen-independent prostate cancer cells. Prostate Cancer Prostatic Dis 3: 84-93, 2000.

13. Hatcher H, Planalp R, Cho J, Torti FM and Torti SV: Curcumin: from ancient medicine to current clinical trials. Cell Mol Life Sci 65: 1631-1652, 2008

14. Shehzad A, Wahid F and Lee YS: Curcumin in cancer chemoprevention: molecular targets, pharmacokinetics, bioavailability, and clinical trials. Arch Pharm (Weinheim) 343: 489-499, 2010.

15. Beevers CS, Chen L, Liu L, Luo Y, Webster NJ and Huang S: Curcumin disrupts the Mammalian target of rapamycin-raptor complex. Cancer Res 69: 1000-1008, 2009.

16. Cuendet $\mathrm{M}$ and Pezzuto JM: The role of cyclooxygenase and lipoxygenase in cancer chemoprevention. Drug Metabol Drug Interact 17: 109-157, 2000.

17. Rao CV: Regulation of COX and LOX by curcumin. Adv Exp Med Biol 595: 213-226, 2007.

18. Verderio P, Bonetti P, Colombo M, Pandolfi L and Prosperi D: Intracellular drug release from curcumin-loaded PLGA nanoparticlesinduces $\mathrm{G} 2 / \mathrm{M}$ block in breast cancer cells. Biomacromolecules 14: 672-682, 2013.

19. Zlotogorski A, Dayan A, Dayan D, Chaushu G, Salo T and Vered M: Nutraceuticals as new treatment approaches for oral cancer - I: Curcumin. Oral Oncol 49: 187-191, 2013.

20. Trott $\mathrm{O}$ and Olson AJ: AutoDock Vina: improving the speed and accuracy of docking with a new scoring function, efficient optimization, and multithreading. J Comput Chem 31: 455-461, 2010.

21. Gallego O, Ruiz FX, Ardèvol A, et al: Structural basis for the high all-trans-retinaldehyde reductase activity of the tumor marker AKR1B10. Proc Natl Acad Sci USA 104: 20764-20769, 2007.

22. Liu KK, Bagrodia S, Bailey S, et al: 4-methylpteridinones as orally active and selective PI3K/mTOR dual inhibitors. Bioorg Med Chem Lett 20: 6096-6099, 2010.

23. Benes CH, Wu N, Elia AE, Dharia T, Cantley LC and Soltoff SP: The C2 domain of PKCdelta is a phosphotyrosine binding domain. Cell 121: 271-280, 2005.

24. Tochowicz A, Maskos K, Huber R, et al: Crystal structures of MMP-9 complexes with five inhibitors: contribution of the flexible Arg424 side-chain to selectivity. J Mol Biol 371: 989-1006, 2007.

25. Wang JL, Limburg D, Graneto MJ, et al: The novel benzopyran class of selective cyclooxygenase-2 inhibitors. Part 2: the second clinical candidate having a shorter and favorable human half-life. Bioorg Med Chem Lett 20: 7159-7163, 2010.

26. Yun CH, Boggon TJ, Li Y, et al: Structures of lung cancer-derived EGFR mutants and inhibitor complexes: mechanism of activation and insights into differential inhibitor sensitivity. Cancer Cell 11: 217-227, 2007.

27. Bikádi Z, Hazai E, ZsilaF and Lockwood SF: Molecular modeling of non-covalent binding of homochiral (3S,3'S)-astaxanthin to matrix metalloproteinase-13 (MMP-13). Bioorg Med Chem 14: 5451-5458, 2006

28. D'hoedt D and Bertrand D: Nicotinic acetylcholine receptors: an overview on drug discovery. Expert Opin Ther Targets 13: 395-411, 2009.

29. Hetényi C and van der Spoel D: Blind docking of drug-sized compounds to proteins with up to a thousand residues. FEBS Lett 580: 1447-1450, 2006.

30. Iorga B, Herlem D, Barré E and Guillou C: Acetylcholine nicotinic receptors: finding the putative binding site of allosteric modulators using the "blind docking" approach. J Mol Model 12: 366-372, 2006

31. Seeliger D and de Groot BL: Ligand docking and binding site analysis with PyMOL and Autodock/Vina. J Computb Aided Mol Des 24: 417-422, 2010

32. DeLano WL: The case for open-source software in drug discovery. Drug Discov Today 10: 213-217, 2005.

33. Matsunaga T, Endo S, Soda M, et al: Potent and selective inhibition of the tumor marker AKR1B10 by bisdemethoxycurcumin: probing the active site of the enzyme with molecular modeling and site-directed mutagenesis. Biochem Biophys Res Commun 389: 128-132, 2009. 
34. Balendiran GK: Fibrates in the chemical action of daunorubicin Curr Cancer Drug Targets 9: 366-369, 2009.

35. Xuyu Z, Ruilan Y, Jun M, Duan-Fang L and Deliang C: AKR1B10: A potential target for cancer therapy. Bioscience Hypotheses 2: 31-33, 2009.

36. Tokunaga $\mathrm{C}$, Yoshino $\mathrm{K}$ and Yonezawa $\mathrm{K}$ : mTOR integrates amino acid- and energy-sensing pathways. Biochem Biophys Res Commun 313: 443-446, 2004.

37. Beevers CS, Zhou H and Huang S: Hitting the golden TORget: curcumin's effects on mTOR signaling. Anticancer Agents Med Chem Dec 2012 (epub ahead of print).

38. Deters M, Hütten H and Kaever V: Synergistic immunosuppressive effects of the mTOR inhibitor sirolimus and the phytochemical curcumin. Phytomedicine 20: 120-123, 2013.

39. Lin JK: Molecular targets of curcumin. Adv Exp Med Biol 595: 227-243, 2007.

40. Lou JR, Zhang XX, Zheng J and Ding WQ: Transient metals enhance cytotoxicity of curcumin: potential involvement of the NF-kappaB and mTOR signaling pathways. Anticancer Res 30 : 3249-3255, 2010

41. Conboy L, Foley AG, O'Boyle NM, et al: Curcumin-induced degradation of PKC delta is associated with enhanced dentate NCAM PSA expression and spatial learning in adult and aged Wistar rats. Biochem Pharmacol 77: 1254-1265, 2009.

42. Tartour E, Pere H, Maillere B, et al: Angiogenesis and immunity: a bidirectional link potentially relevant for the monitoring of antiangiogenic therapy and the development of novel therapeutic combination with immunotherapy. Cancer Metastasis Rev 30: 83-95, 2011.

43. van Kempen LC and Coussens LM: MMP9 potentiates pulmonary metastasis formation. Cancer Cell 2: 251-252, 2002

44. Bolignano D, Donato V, Lacquaniti A, et al: Neutrophil gelatinase-associated lipocalin (NGAL) in human neoplasias: a new protein enters the scene. Cancer Lett 288: 10-16, 2010

45. Masson V, de la Ballina LR, Munaut C, et al: Contribution of host MMP-2 and MMP-9 to promote tumor vascularization and invasion of malignant keratinocytes. FASEB J 19: 234-236, 2005.

46. Jankun J, Aleem AM, Selman SH, Basrur V and Skrzypczak-Jankun E: VLHL plasminogen activator inhibitor spontaneously reactivates from the latent to active form. Int J Mol Med 23: 57-63, 2009.

47. Jankun J, Aleem AM, Specht Z, et al: PAI-1 induces cell detachment, downregulates nucleophosmin (B23) and fortilin (TCTP) in LnCAP prostate cancer cells. Int J Mol Med 20: 11-20, 2007.
48. Jankun J and Skrzypczak-Jankun E: Yin and yang of the plasminogen activator inhibitor. Pol Arch Med Wewn 119: 410-417, 2009.

49. Ravindranath $\mathrm{MH}$, Muthugounder S, Presser $\mathrm{N}$ and Viswanathan S: Anticancer therapeutic potential of soy isoflavone, genistein. Adv Exp Med Biol 546: 121-165, 2004.

50. Fujisawa S, Atsumi T, Ishihara M and Kadoma Y: Cytotoxicity, ROS-generation activity and radical-scavenging activity of curcumin and related compounds. Anticancer Res 24: 563-569, 2004.

51. Youssef KM, El-Sherbeny MA, El-Shafie FS, Farag HA, Al-Deeb OA and Awadalla SA: Synthesis of curcumin analogues as potential antioxidant, cancer chemopreventive agents. Arch Pharm (Weinheim) 337: 42-54, 2004.

52. Zingg JM, Hasan ST and Meydani M: Molecular mechanisms of hypolipidemic effects of curcumin. Biofactors 39: 101-121, 2013.

53. Menon VP and Sudheer AR: Antioxidant and anti-inflammatory properties of curcumin. Adv Exp Med Biol 595: 105-125, 2007.

54. Gafner S, Lee SK, Cuendet M, et al: Biologic evaluation of curcumin and structural derivatives in cancer chemoprevention model systems. Phytochemistry 65: 2849-2859, 2004.

55. Guo LY, Cai XF, Lee JJ, et al: Comparison of suppressive effects of demethoxycurcumin and bisdemethoxycurcumin on expressions of inflammatory mediators in vitro and in vivo. Arch Pharm Res 31: 490-496, 2008.

56. Handler N, Jaeger W, Puschacher H, Leisser K and Erker T: Synthesis of novel curcumin analogues and their evaluation as selective cyclooxygenase-1 (COX-1) inhibitors. Chem Pharm Bull (Tokyo) 55: 64-71, 2007.

57. Griesser M, Pistis V, Suzuki T, Tejera N, Pratt DA and Schneider C: Autoxidative and cyclooxygenase-2 catalyzed transformation of the dietary chemopreventive agent curcumin. J Biol Chem 286: 1114-1124, 2011

58. Dong L, Vecchio AJ, Sharma NP, Jurban BJ, Malkowski MG and Smith WL: Human cyclooxygenase-2 is a sequence homodimer that functions as a conformational heterodimer. J Biol Chem 286: 19035-19046, 2011.

59. Xu YY, Cao Y, Ma H, Li HQ and Ao GZ: Design, synthesis and molecular docking of $\alpha, \beta$-unsaturated cyclohexanone analogous of curcumin as potent EGFR inhibitors with antiproliferative activity. Bioorg Med Chem 21: 388-394, 2013.

60. Kolev TM, Velcheva EA, Stamboliyska BA and Spiteller M: DFT and experimental studies of the structure and vibrational spectra of curcumin. Int J Quantum Chem 102: 1069-1079, 2005. 